\title{
Alfabetización científica y tecnológica en las instituciones educativas de básica y media y el desarrollo de pensamiento crítico, lógico y matemático como factor generador de una industria de software en Colombia
}

Scientific and technological literacy in educational institutions of basic and media and the development of critical, logical and mathematical thinking as a generator of a software industry in Colombia

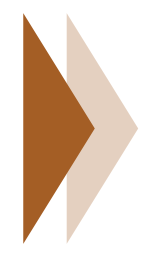

Proyecto de investigación realizado en el marco de la convocatoria 12 del Centro de investigaciones VUAD, Universidad Santo Tomás, perteneciente a la Linea medular: Enrique Lacordie. Línea activa: Organización y Gestión Educativa y del Conocimiento

\section{Floralba Gómez Castaño}

Administradora Educativa y Magister en Pedagogías activas y Desarrollo Humano. Docente CAU Manizales Universidad Santo Tomas.

Correo electrónico: floralbagomezstadistancia.edu.co

\section{Mario Contreras Castro}

Docente Facultad de Ciencias y Tecnologías, Universidad Santo Tomás.

Correo electrónico: mariocontreras@ustadistancia.edu.co 


\section{Pastor Martín Bohorquez}

Ingeniero de sistemas, Especialista en Software para redes, Magister en Educación.

Docente medio tiempo Facultad de Educación. Universidad Santo Tomás.

Correo electrónico: pastormartin@ustadistancia.edu.co

\section{Yadira Ruiz Díaz}

Trabajadora Social, Magister en estudios y Gestión del Desarrollo. Docente CAU

Manizales, Universidad Santo Tomas.

Correo electrónico: yadiraruizstadistancia.edu.co

\section{Resumen}

Este proyecto de investigación reconoce la importancia de identificar, caracterizar los procesos de formación en el área de Tecnología e Informática y el nivel de desarrollo del pensamiento crítico y lógico matemático que suscita en los estudiantes de básica y media de las instituciones educativas ubicadas dentro de las regiones de influencia de los Centros de Atención Universitaria, -CAU- de la Vicerrectoría de Universidad Abierta y a Distancia de la Universidad Santo Tomás

La metodología para el desarrollo del proyecto se enmarca en el enfoque cualitativo, tomando como base el estudio de naturaleza aplicada y de tipo descriptivo, en tanto tiene el propósito de caracterizar y describir sistemáticamente los hechos y características de una población.

En el transcurso de la investigación se retoman documentos nacionales e internacionales: "Standards For Technological Literacy" publicados por el International Technology Education Association ITEA en el 2007, donde se determinan las recomendaciones centrales para la implementación de una Alfabetización Tecnológica; El MEN en su documento "ser competente en tecnología - una necesidad para el desarrollo", también establece las pautas de formación y competencias que se deben desarrollar en el área de "tecnología e informática" en la educación básica y media; igualmente tenidas en cuenta en esta investigación.

Palabras clave: tecnología e informática, problemas, software, lógica de programación, algoritmo, pensamiento crítico, pensamiento lógico matemático

\section{Abstract}

This research project recognizes the importance of identifying and characterizing the training processes in the area of Technology and Informatics that are developed in the educational institutions of basic and secondary education, located within the regions of influence of the CAU of the University Vice-Rector's Office Open and away from Santo Tomas University.

The project development methodology is framed in the qualitative approach, based on the study of an applied and descriptive nature, in order to systematically characterize and describe the facts and characteristics of a population.

In the course of the research, base documents such as the Standards for Technological Literacy published by the International Technology Edu- 
cation Association (ITEA) in 2007 were retaken, where it established the central recommendations for the implementation of a technological Literacy. In the national framework, the document "Being competent in technology - A need for development" is taken up again. These standards established the training guidelines and competences to be developed in the area of "Technology and Informatics" in basic and secondary education.

Keywords: technology and computer science, problems, software, programming logic, algorithm, critical thinking, mathematical logical thinking

\section{Contextualización de la investigación}

En el documento titulado "Colombia: al filo de la oportunidad" publicado en 1996, se puntualiza la necesidad de abordar la ciencia y la tecnología en el sistema educativo, su importancia en el crecimiento económico y el desarrollo de nuestro país. Esto permite pensar que Colombia necesita unos lineamientos nuevos en su sistema educativo para que los modelos pedagógicos y gestiones curriculares de las instituciones educativas, apunten al fomento y desarrollo de habilidades científicas y tecnológicas; así como culturales y socioeconómicas.

Este planteamiento, convoca a una reestructuración conceptual y organizativa, una reorientación del imaginario colectivo y la generación de nuevos valores, comportamientos, aptitudes cognitivas y prácticas organizacionales adaptadas al mundo moderno.

En el informe (Colombia al Filo de la Oportunidad) llama la atención sobre las deficiencias en nuestro sistema educativo respecto a la enseñanza de la ciencia, la tecnología, su proyección y la carencias de capital humano capacitado; sistemas educativos de calidad con amplia cobertura y la inadecuada educación científica para el desarrollo tecnológico de Colombia (COLCIENCIAS, 2016). Rodolfo Llinas, coautor de este informe, afirmó:

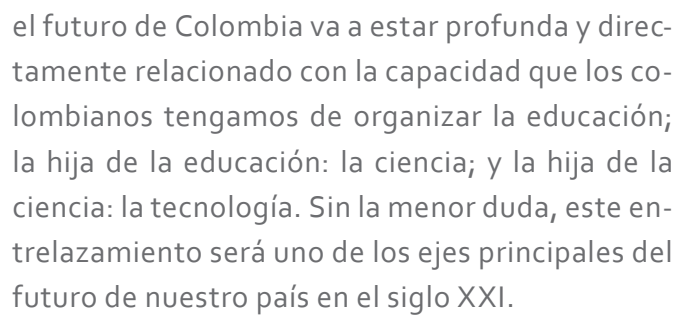

Actualmente una de las tecnologías que ha marcado el desarrollo en diferentes áreas económicas e industriales, es la producción y manufacturación de Software. La revista Dinero publicó en 2011 cifras del informe de PwC, "Global 100 Software Leaders, Key Players \& Market trends. Líderes mundiales, principales actores y tendencias del mercado del software", donde se pudo identificar con certeza, que el mercado del software, estaba aproximadamente en una cifra de US $\$ 250.000$ millones; es así que esta industria contribuye a la productividad y crecimiento general de la economía global, debido a los altos niveles de competitividad e innovación que lleva a las otras industrias. Adicionalmente, esta publicación menciona que los países líderes en la industria del software son China, Francia, Alemania, India, el Reino Unido y los Estados Unidos (Dinero, 2016).

Zuluaga (2011) a través del "estudio del comportamiento de la industria del software en Colombia ante escenarios de capacidades de innovación y ventajas comparativas por medio de dinámica de sistemas", identificó que en este campo tecnológico nuestro país tiene un serio problema para desarrollar esta industria, ya que el número de graduados de ingeniería de sistemas ha caído un $5 \%$ anual, esta cifra es baja sí se compara con otros países como China, India, Corea y Brasil; países que han mostrado incrementos del $26 \%$ anual de graduados, en el caso de China, y del $10 \%$ para el caso de Brasil. La situación descrita anteriormente, refleja una crisis o problemática en los planes de formación específica del recurso humano necesario para el desarrollo de esta industria en Colombia. 
Por tanto, es evidente e imperante trazar metas y desafíos transformadores en los procesos educativos, para abordar y apropiar en los currículos una alfabetización científica y tecnológica, que logre generar ambientes de aprendizaje para su contextualización y comprensión, que apunte a despertar mentes innovadoras capaces de transformar sus entornos, descubrir y aplicar soluciones a necesidades sociales directas y reales, de tal manera que generen bienestar y desarrollo en las diferentes regiones de nuestro país. La creación de dichas condiciones permitiría la formación de un talento humano sintonizado con las competencias requeridas en este nuevo siglo, por ejemplo, la de competencias en pensamiento lógico matemático: el cual es necesario en la construcción algorítmica dentro de la industria del software (Palomino, 2016).

Adrián F. Ruffinatti (2016), en su artículo "La industria del software en la india: ¿un éxito casual?", presenta un acercamiento de la estrategia seguida por este país para ser uno de los líderes en proveer al mundo, de recursos humanos especializados en la construcción de soluciones de software. El autor resalta que uno de los aspectos que es factor de éxito en la India, es el contar con alrededor de 300.000 profesionales calificados trabajando en la industria de las tecnologías de la información; menciona, que casi 100.000 ingenieros en software se gradúan al año, aportando una fuente de conocimientos inagotable para la industria local, ya que estos jóvenes se han inclinado hacia una especialización informática debido a un fuerte nivel de conocimiento previo en matemáticas, idiomas y lógica, competencias necesarias de un ingeniero de software.

Es por tanto, que realizar procesos de transformación en el sistema educativo para abordar una alfabetización científica y tecnológica que logre generar ambientes de aprendizaje y faciliten su contextualización y comprensión para despertar mentes innovadoras, transformadoras de sus entornos y solucionen necesidades directas generando bienestar y desarrollo en las diferentes regiones de nuestro país, permite desarrollar un recurso humano formado con las competencias requeridas en este nuevo siglo, por ejemplo, la de competencias en pensamiento crítico, lógico y matemático, necesarias en la construcción algorítmica dentro de la industria del Software (Palomino, 2016).

Estos antecedentes interpelan y validan procesos de investigación que conducen a preguntar: ¿qué procesos de alfabetización científica y tecnológica promueven el desarrollo de pensamiento crítico, lógico y matemático, claves dentro de la ingeniería de software, en las instituciones educativas de formación básica y media, ubicadas en las regiones de influencia de los $\mathrm{CAU}^{1}$ de la USTA ${ }^{2}$ ?

El International Technology Education Association ITEA publicó en el 2007 la tercera edición del documento titulado "Standards for technological literacy" donde se establecieron las recomendaciones centrales para la implementación de una Alfabetización Tecnológica y se conceptualizó ésta como: "la habilidad para usar, administrar, evaluar y entender la tecnología" además se puntualiza que "la tecnología es la transformación de un ambiente natural con el fin de satisfacer una necesidad o deseo humano" (Association, 2018).

La palabra griega techné, que significa el arte o artificio o artesanía, indica que el término tecnología, literalmente, significa el acto de realizar o hacer a mano, pero más generalmente se refiere a la colección de procesos y conocimiento que la gente usa para ampliar las capacidades humanas (Association, 2016).

La ITEA es incisiva respecto a la diferencia entre educación en tecnológica y tecnología educativa, plantea que la tecnología educativa hace referencia a "la utilización de la tecnología como una herramienta para mejorar el proceso de enseñanza - aprendizaje" (ACM, 2018). La educación en tecnología, en cambio, tiende a la organización de

1. CAU: Sigla que da la Vicerrectoría de Universidad Abierta y a distancia de la Universidad Santo Tomás a los Centro de Atención Universitaria, que se encuentran en 23 ciudades de Colombia.

2. USTA: Sigla que se da a la Universidad Santo Tomas Colombia. 
los ambientes escolares, para que los estudiantes aprendan acerca el mundo tecnológico que los inventores, ingenieros y otros innovadores han creado. Estudian cómo se genera energía a partir de carbón, gas natural, energía nuclear, energía solar, y el viento, y cómo se transmite y se distribuye, entre otros contenidos.

El estudiante examina los sistemas de comunicación: teléfono, radio y televisión, comunicaciones por satélite, fibra óptica, internet, entre otros. Profundizan en las técnicas de fabricación, los materiales y los procesos industriales. Investiga e indaga sobre nuevas tecnologías aplicables a: el transporte, el procesamiento de información y la tecnología médica. El objetivo es que el escolar adquiera una mayor capacidad para la comprensión conceptual, sobre la importancia de la relación de la ciencia y la tecnología y su lugar en la sociedad (CTS). Por tanto, la alfabetización tecnológica hace hincapié en la comprensión de los elementos básicos que intervienen en cualquier tecnología, por ejemplo: - el proceso de diseño, que es el enfoque principal de ingenieros, diseñadores y productores en la utilización de la tecnología para la creación de soluciones a los problemas. - el desarrollo y la producción, donde el diseño le da el acabado al producto y se crea un sistema o proceso que se desarrolla para la producción.

La esencia o naturaleza misma de la alfabetización tecnológica estimula el desarrollo de habilidades de pensamiento y facilita la utilización de herramientas mentales, promueve procesos de pensamiento orientados a la secuencia lógica de hallazgos, en procura de soluciones que transformen los entornos en los cuales está inmerso el estudiante. En consecuencia, el estudiante podrá entender la tecnología como la expresión práctica de la ciencia.

Los "Standards for technological literacy", consideran que una de las grandes ventajas de aprender acerca de la tecnología es que privilegia procesos de aprendizaje experimental; lo cual garantiza aprendizajes activos y significativos. Una persona alfabetizada tecnológicamente, entiende lo que la tecnología es, cómo se crea y cómo influencia la sociedad, y a su vez como está es determinada por la sociedad.

Conocer y comprender la dinámica interna de los procesos de alfabetización en tecnología, permite hacer una lectura crítica y en consecuencia plantear nuevas formas de abordarla para fortalecerla o mejorarla. Cuando no se hace seguimiento, no se cuestiona continuamente este proceso alfabetizador, se obstaculiza la posibilidad de revisar la coherencia de la tecnología con el mundo de necesidades sociales, el cambio continúo y entender ese decodificador interno que posee y que hace repensarla en forma constante.

En el año 2008 el Ministerio de Educación Nacional publicó las orientaciones generales para la Educación en Tecnología e Informática, en una cartilla denominada "Ser competente en tecnología. Una necesidad para el desarrollo". Estos estándares establecieron las pautas de formación y las competencias que se debían desarrollar en el área de "tecnología e informática" en la educación básica y media (MEN, 2016). Desde los siguientes componentes de formación:

- Naturaleza y evolución de la tecnología.

- Apropiación y uso de la tecnología.

- Solución de problemas con tecnología.

- Tecnología y sociedad.

De igual manera, define las "competencias" como un conjunto de conocimientos, habilidades, actitudes, comprensiones y disposiciones cognitivas, meta cognitiva, socio afectivas y psicomotoras; por ejemplo

- Reconozco y describo la importancia de algunos artefactos en el desarrollo de actividades cotidianas en mi entorno y en el de mis antepasados.

- Reconozco productos tecnológicos de entorno cotidiano y los utilizo en forma segura y apropiada. (Ministerio de Educación Nacional, 2016). 
Asimismo, el Ministerio de Educación Nacional (2016) estableció que los "desempeños" para reconocer el desarrollo de la competencia en los estudiantes, fueran asumidos como señales o pistas que ayudan al docente a valorar la competencia en sus estudiantes. Contienen elementos, conocimientos, acciones, destrezas o actitudes deseables para alcanzar la competencia propuesta.

Estos estándares se establecieron para los siguientes grupos de formación:

- de primero a tercero

- de cuarto a quinto

- de sexto a séptimo

- de octavo a noveno y

- de décimo a undécimo

En consecuencia, esta investigación buscó identificar cómo se está comprendiendo en las instituciones educativas estos lineamientos; si realmente están logrando una alfabetización tecnológica que logre en los estudiantes procesos integradores entre la ciencia, la tecnología y las competencias en el desarrollo de software. Así que la Universidad Santo Tomás, en su modalidad abierta y a distancia, a través de los programas de ingeniería informática y licenciatura en tecnología e informática, se vincula, visiona y promueve la investigación educativa; desde la base de formación, los niveles de educación básica primaria, secundaria y media, la posibilidad de fundamentar teórica y pedagógicamente una nueva estructura de lo que debe ser la educación en tecnología, y apuntarle a proyectos desde la regiones que puedan dar una trascendencia en el desarrollo tecnológico e informática del país, especialmente el desarrollo de Software.

\section{Metodología}

Dada la naturaleza del estudio, el proceso metodológico del proyecto se realizó desde un enfoque cualitativo, por cuanto tiene el propósito de caracterizar y describir sistemáticamente los hechos y características de una población (Hernández, Fer- nández \& Baptista, 1994), para esta investigación, eran las instituciones educativas de básica primaria, secundaria y media, donde los CAU regionales tenían influencia.

La metodología comprendió tres grandes fases: preparatoria, interpretativa, propositiva. A continuación, se describe el proceso:

- Conceptualización desde los "Standards for Technological Literacy" y los estándares del Ministerio de Educación Nacional, en relación con la Educación en Tecnología e Informática.

- Recolección de información que condujera a identificar dentro de la gestión curricular de las instituciones educativas, las formas o estrategias como están implementadas las áreas de matemáticas, tecnología e informática; con el fin de encontrar cómo se promueve el desarrollo del pensamiento crítico, lógico y matemático; fundamental para la formación de futuros ingenieros de software.

- Recolección de información para identificar las principales estrategias didácticas que aplican las instituciones de educación básica primaria, secundaria y media, para desarrollar las competencias propias del área de matemáticas, de tecnología e informática y las estrategias implementadas para el desarrollo de habilidades de pensamiento.

- Recolección de información para establecer los imaginarios, representaciones mentales y preconceptos de los docentes y directivos de estas instituciones educativas, con respecto a la tecnología e informática como disciplina y su función mediadora en procesos educativos.

- Contrastación de la visión que tienen actualmente los docentes y directivos con respecto a los fines de formación del área de matemática y los establecidos en los estándares definidos por el MEN. 
Para los puntos 2, 3, 4 y 5 de la educación, se aplicaron instrumentos de recolección de datos que permitiera identificar los conceptos que tienen los docentes y directivos de los fines de formación de las áreas de matemáticas y de tecnología e informática.

\section{Resultados}

Se aplicaron 61 fichas de resumen analítico especializado (RAE), en Instituciones de 40 ciudades y/o municipios colombianos: Aguazul (Casanare), Baraya (Huila), Barrancas (La Guajira), Bogotá D.C. (Cundinamarca), Bucaramanga (Santander), Carmen de Carupa, (Cundinamarca), Carmen Del Chucuri
(Santander), Chía (Cundinamarca), Chitagá (Santander), Cogua (Cundinamarca), Cúcuta (Norte De Santander), Dorada (Caldas), El Peñón (Santander), Espinal (Tolima), Funza (Cundinamarca), Fusagasugá (Cundinamarca), Granada (Meta), Lejanías (Meta), Lorica (Córdoba), Manizales (Caldas), Medellín (Antioquia), Medina (Cundinamarca), Montería (Córdoba), Mosquera (Cundinamarca), Nocaima (Cundinamarca), Palmitos (Sucre), Pasto (Nariño), Popayán (Cauca), Resguardo Indígena de Quichaya Municipio de Silvia (Cauca), Rivera (Huila), Soacha (Cundinamarca), Tunja (Boyacá), Ubaté (Cundinamarca), Valledupar (Cesar), Vereda Bojacá (Chía), Vereda Palo Bajito (Yopal, Casanare), Vereda San Mateo (Bucaramanga), Villa De Leiva (Boyacá), Villapinzón (Cundinamarca), Villavicencio (Meta) y Zapatoca (Santander).

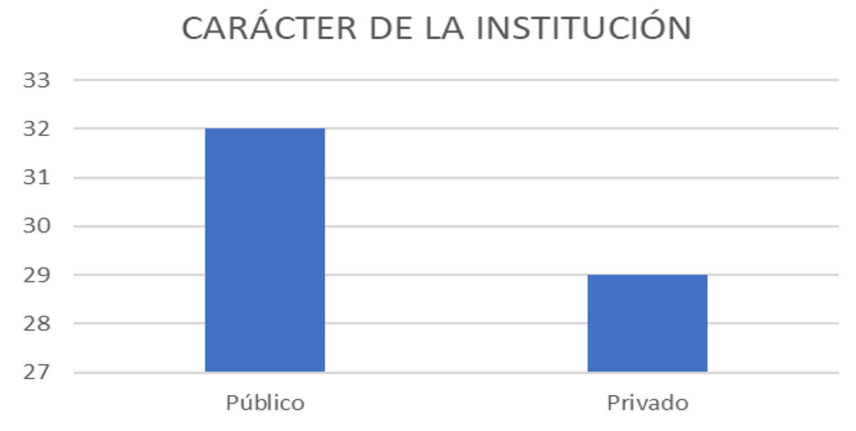

Gráfico 1. Carácter de la institución.

De las 61 instituciones 32 de ellas son de carácter público y 29 de carácter privado.
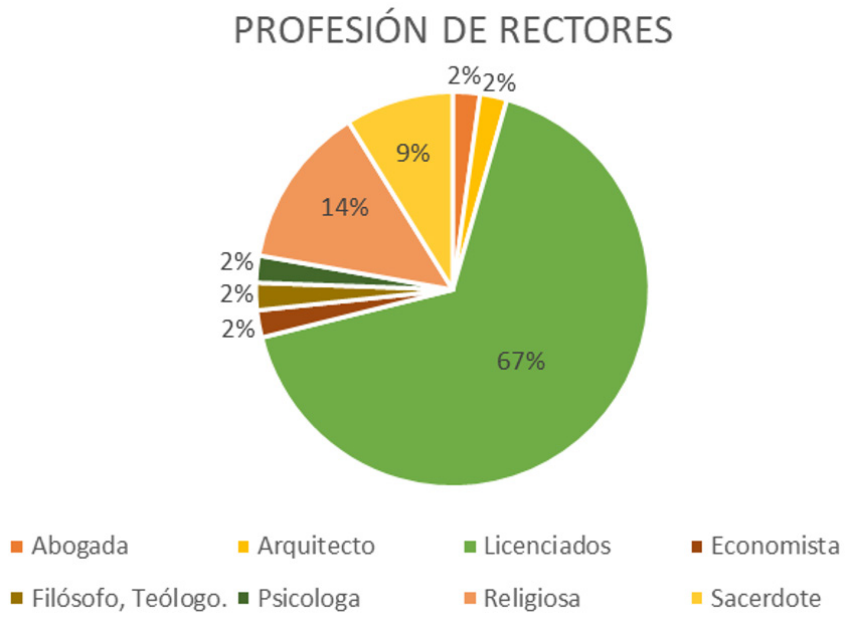

Gráfico 2. Profesión rectores. 
Frente a la formación de los rectores se obtuvo información de 45 de ellos, encontrando que: un $60 \%$ de los rectores de las instituciones educativas son licenciados, en áreas afines a la administración educativa, seguido por religiosos y sacerdotes, y una misma proporción otras carreras como economistas, filósofos, teólogos, arquitectos, psicólogos y abogados.

\section{Profesión docentes matemáticas}

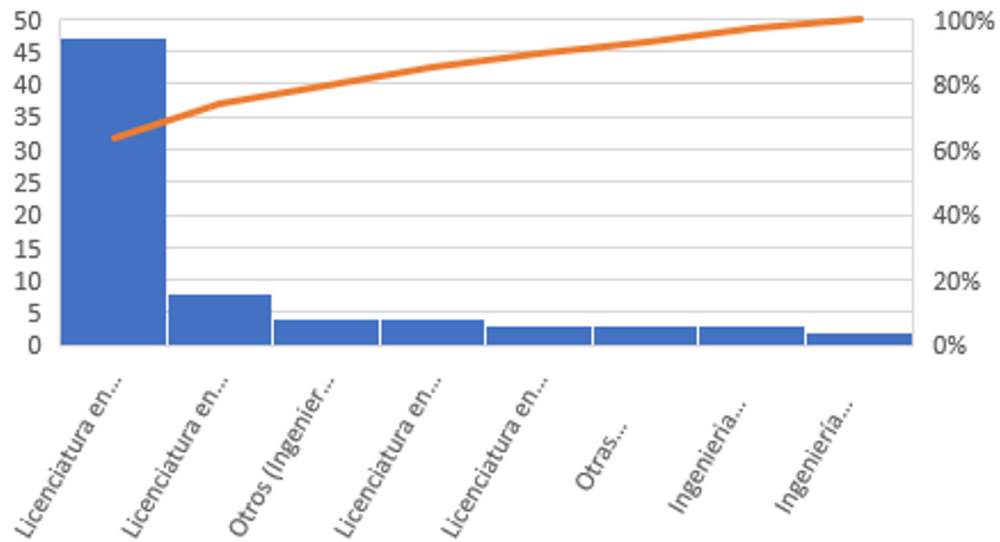

Gráfico 3. Profesión docentes de matemáticas.

En cuanto a la profesión de los docentes de matemáticas se encontró un: 90\% son licenciados en matemáticas, seguidamente con un 15\% licenciados en física y matemáticas, y con un $9 \%$ otras carreras como ingeniería metalúrgica, ingeniería de sistemas, administración de empresas y educadores especiales. Por último, con porcentajes bajos licenciatura en básica, ingeniería química, otras licenciaturas como licenciatura en biología y química, licenciatura en física, licenciatura en educación infantil. Estos datos evidencian que de cierta manera se cumple con el perfil propio de un docente de matemáticas.

Profesión docentes en tecnología e informática

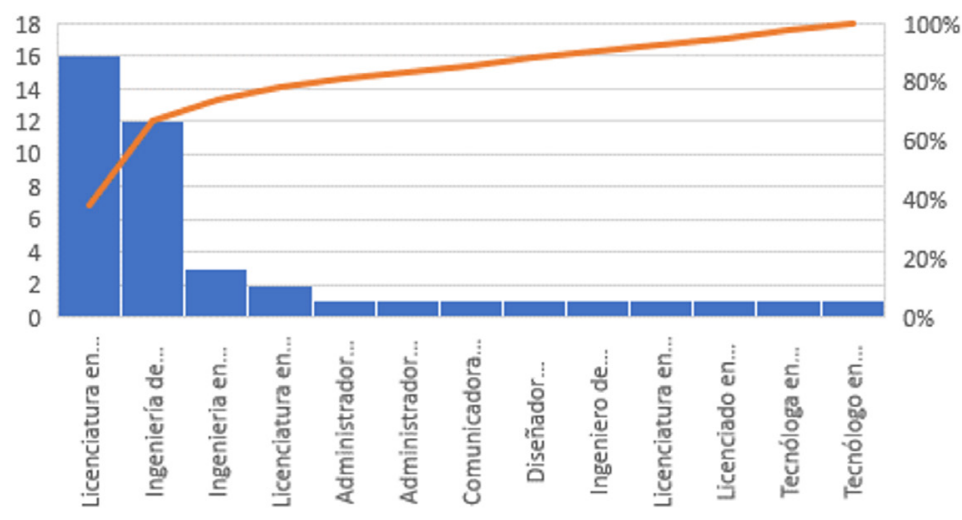

Gráfico 4. Profesión docentes de Tecnología e informática. 
Frente a la profesión de los docentes de tecnología e informática, se estableció que el $90 \%$ son licenciados en informática y tecnología, seguido de ingenieros de sistemas con un $70 \%$ (los cuales, si bien poseen el conocimiento en tecnología, no cumplen con el perfil de docentes), posteriormente en bajos porcentajes ingenieros de petróleos, licenciados en educación física, licenciados en educación básica, licenciados en electrónica e ingeniero de sistemas, tecnóloga en agronomía y tecnólogo en sistemas. En los dos últimos casos es preocupante, ya que, los docentes no cumplen con el mínimo de estudios para el ejercicio docente.

A diferencia de los docentes de matemática, que en gran porcentaje cumplen con los perfiles propios de esa asignatura, los docentes de tecnología e informática no cumplen dicho perfil y se relacionan con otras carreras.

De acuerdo con la categoría de propuesta pedagógica, la información mostró que las instituciones educativas, trabajan desde un modelo pedagógico definido con un enfoque pedagógico determinado por dicho modelo. Encontrando en los más comunes los siguientes:

- Modelo Pedagógico Cognitivista con enfoque constructivista; busca que el estudiante y el maestro construyan juntos el conocimiento desde la interacción con el medio, las informaciones teóricas y la experiencia del estudiante; sustenta que es el estudiante quien elabora su conocimiento cuando interactúa con el objeto del conocimiento y con los otros en un contexto significativo.

- Modelo Pedagógico Relacional Sistemático con un enfoque investigativo; el cual busca a través de la pedagogía, organizar y desarrollar procesos educativos articulando las dimensiones psicológica, física, intelectual, cultural y social, de los estudiantes para desarrollar competencias investigativas; trabaja sobre el principio que la interacción del estudiante con la indagación y las preguntas sobre el mundo, favorecen el conocimiento y por lo tanto su desarrollo integral.
- Modelo Pedagógico Cognitivista con un enfoque humano, que apunta al desarrollo de competencias ciudadanas para Formar los ciudadanos que la sociedad colombiana necesita; un ser humano que aprenda a vivir con los demás y a apropiar los valores para desempeñarse adecuadamente en la sociedad; con competencias que le ayuden a actuar con eficiencia y demostrando habilidades y destrezas en la solución de problemáticas que se presenten en su contexto en su realidad cotidiana.

- Modelo Pedagógico "Escuela Activa" con enfoque constructivista como una propuesta educativa abierta y flexible, dirigida a la formación integral de los estudiantes; el maestro y el joven construyen juntos el conocimiento desde los saberes previos, caracterizado por el trabajo en equipo, aprendizaje colaborativo y el protagonismo de los estudiantes en su propio aprendizaje; busca formar personas capaces de profundizar los conocimientos indagando en otras fuentes y aplicando las tecnologías de la información y la comunicación.

Según la categoría metodología para la enseñanza de las matemáticas y la tecnología e informática, las instituciones educativas responden a unas estrategias de enseñanza que son generales o comunes en todas las respuestas; puede decirse que prevalece la retroalimentación de los conocimientos construidos, la exploración de los saberes previos de los estudiantes respecto a una temática, los trabajos de indagación, producción y profundización de objetos del conocimiento en equipos, el uso de guías de inter aprendizaje, talleres instructivos y las socializaciones, como muestra de la apropiación del conocimiento; puede decirse que se utiliza una metodología inductiva - deductiva.

Otra de las categorías analizadas fue la evaluación; se quiso indagar sobre el sistema de evaluación que acogen las instituciones, para determinar los avances o retrocesos en el desarrollo de las competencias pertinentes a las matemáticas y la tecnología e informática; por lo tanto, se encuentra que para todas los centros educativos donde 
se aplicó el RAE (regidos por los lineamientos del decreto 1290 de 16 de abril de 2009), conciben la evaluación como un proceso de revisión continuo y permanente que determina una oportunidad para que el estudiante demuestre sus saberes. El análisis muestra que las estrategias de evaluación más utilizadas son la autoevaluación, la coevaluación, la heteroevaluación, los mapas mentales como instrumento de apropiación de conceptos; las evaluaciones escritas y las sustentaciones de trabajos que deben hacer los estudiantes para demostrar saberes; estas valoraciones se realizan de acuerdo a la escala de valoración que trae el decreto antes mencionados y son registradas en listados llamadas "planillas de notas".

Respecto a la categoría de concepto de alfabetización tecnológica, sobre la cual trabajan las instituciones, se encontró que cada centro educativo, concibe de manera particular la enseñanza de la tecnología e informática, no obstante coinciden en que es un área fundamental, para la adquisición del conocimiento y la proyección profesional de sus estudiantes; sin embargo, se encontraron algunos conceptos similares como que la alfabetización en tecnología e informática se refiere al "ritmo de expansión y cambio del conocimiento que demanda una mayor articulación e interdependencia, entre el conocimiento empírico y el conocimiento técnico y científico" ; a la capacidad para aprender a innovar y construir con mayor facilidad el conocimiento; "como la ciencia dinámica y en constante evolución, con amplio margen para la intuición y la creatividad". Es decir, una forma de producción y desarrollo de pensamiento para explicar, interpretar y utilizar en la resolución de problemas. Todo parece indicar que conciben la alfabetización tecnológica como un mediador para la construcción del conocimiento y la futura profesionalización de sus estudiantes.

Las competencias en tecnología e informática son también una categoría analizada desde lo que cada institución plantea en su plan curricular. A este respecto, se encontró que en el nivel de la básica primaria se busca el desarrollo de capacidades para comprender, interpretar y proponer sobre la naturaleza y evolución de la tecnología; la importancia en el contexto actual y la necesidad de usar esas tecnologías con responsabilidad. Las competencias en el nivel de la básica secundaria muestran que se trata de desarrollar capacidades para la correcta exploración, utilización e innovación de la tecnología y la informática; en la educación media las competencias están dirigidas a la comprensión de la articulación de la ciencia, la tecnología y sociedad, muestra este estudio que los estudiantes desarrollan capacidades para comprender que el conocimiento, permite generar nuevas ideas que se hacen realidad en nuevos artefactos, procesos o producciones. Estas competencias también están enfocadas a la apropiación y el aprovechamiento para el mejoramiento en el desarrollo de las propias producciones académicas de los estudiantes.

Finalmente, se evidencia que, en la propuesta curricular de las instituciones, están reflejadas las competencias de matemáticas, tecnología e informática establecidas por el Ministerio de Educación Nacional y se atiende las recomendaciones del Ministerio de las TIC, en el sentido de ajustar esas competencias al contexto y la cultura de las comunidades. Asimismo, los contenidos para estas dos áreas, se derivan de los estándares que determina el Ministerio de Educación; es decir, competencias y contenidos no son diferentes a los que son emanados del MEN; se pueden visibilizar en mallas curriculares institucionales que organizan estos contenidos y competencias.

En cuanto al análisis realizado a las percepciones que tienen los docentes frente a los lineamientos establecidos por el MEN, se encuentra que no conciben claramente dichos lineamientos, contrastado esto con la categoría de estrategias de articulación, no existe ninguna relación entre lo propuesto en los proyectos educativos institucionales, con una verdadera estrategia de articulación, entre el área de matemática, ni ninguna de las otras áreas del saber a trabajar en los currículos. 


\section{Conclusión}

La investigación logró establecer que el nivel desarrollo de los estándares de educación en tecnología del Ministerio de Educación Nacional, se cumplen en términos de contenidos y desarrollo de competencias, no obstante, la articulación del área de tecnología e informática con otra área o ambientes, no se establece como prioridad en los centros educativos.

Desde los hallazgos de la presente investigación se puede afirmar:

- La alfabetización en tecnología e informática es un proceso de enseñanza aprendizaje que se gestiona y lidera en todas las instituciones educativas, cuyo contenido hace parte de los planes curriculares de cada establecimiento de educación básica y media.

- La tecnología e informática es un área del saber fundamental, cuyos lineamientos curriculares son emanados del Ministerio de Educación Nacional y son de obligatoria planeación, ejecución y evaluación, por lo tanto, se encuentra en todas las propuestas curriculares de las instituciones educativas participantes de este estudio.

- Los estándares de competencias y contenidos sobre tecnología e informática, determinados por el MEN y avalados por el Ministerio de las TIC en Colombia, se identifican y relacionen con los estándares internacionales, ya que se puede evidenciar que la naturaleza, intencionalidades y fines de éstos, corresponden a lo que Colombia busca para la alfabetización tecnológica en los distintos niveles educativos.

- Cada institución educativa en cuestión, presenta un modelo pedagógico cuyo enfoque señala un concepto particular sobre "alfabetización tecnológica", lo cual indica que la gestión académica incluye esta área en su propuesta curricular.
- De manera general, las instituciones educativas conciben la alfabetización tecnológica como un proceso de aprendizaje que potencia la creatividad, desarrolla el pensamiento y estimula la capacidad de innovación de los estudiantes.

- Las instituciones educativas no conciben su proceso de educación, sin la alfabetización en tecnología, pues consideran que esta es la mediación para el desarrollo las competencias y habilidades, que necesita un joven para un buen desempeño en el futuro profesional.

- La metodología utilizada para la enseñanza y aprendizaje de la tecnología e informática, corresponde al "método inductivo"; esto significa que se desarrollan habilidades de pensamiento como la observación, comparación, razonamiento, reflexión y generalización entre otras.

- La alfabetización en tecnología e informática es un área del saber que se evalúa de acuerdo a los lineamientos legales establecidos por el MEN, cuyas estrategias de seguimiento se ven reflejadas en los sistemas de evaluación institucionales, en los que se plantean autoevaluaciones, coevaluaciones, hetero-evaluaciones y ferias de la ciencia en las que los estudiantes muestran sus aprendizajes; todo indica que la evaluación del proceso de alfabetización en tecnología e informática es una estrategia que mide el desempeño de los estudiantes en ésta área.

- Para la alfabetización en tecnología e informática las instituciones cuentan con la infraestructura y los recursos didácticos, de acuerdo con las capacidades económicas y estructurales de las instituciones educativas, es decir, existen los espacios y los materiales.

- El estudio muestra que no se encontraron estrategias de articulación del área de la tecnología e informática, con las matemáticas u otros saberes; esto indica que no existe una transver- 
salización del área con el currículo académico de estas instituciones, por lo tanto, se puede concluir que la alfabetización en tecnología e informática se da como una materia aislada.

- Todas las instituciones educativas han entendido y mostrado que la enseñanza de la Tecnología y la informática son un área que está incluida en el llamado pensum académico; pero no está siendo liderada o administrada pedagógicamente por un profesional con el perfil pertinente a esta área; es decir, los docentes encargados de la alfabetización en tecnología e informática no tienen el perfil profesional correspondiente al área.

La presente investigación deja puntos de partida importantes en donde se debe anclar futuras investigaciones que permitan aflorar propuestas para que se logre asumir el área de tecnología e informática, como área integradora de saberes que viabilice a través del uso de tecnologías del aprendizaje y el conocimiento, desarrollos de procesos de pensamiento lógico y matemático que permitan al país el desarrollo adecuado en ciencia y tecnología. En consecuencia, como se ha mencionado en este artículo, en permitir fortalecer la formación de profesionales en el campo de la ingeniería de software.

\section{Referencias}

Agencia Nacional de Evaluación de la Calidad y Acreditación (2004). Libro blanco de la titulación de la ingeniería en informática. Recuperado de: http://portal.uned.es/pls/portal/docs/ PAGE/UNED_MAIN/LAUNIVERSIDAD/ VICERRECTORADOS/ESPACIOEURO/LIBROS\%20BLANCOS\%20DE\%20GRADO/LIBROBLANCO_TELECOMUNICACIONES. PDF

Association for Computing Machinery-ACM. (2018). About the ACM Organization. Recu- perado de: http://www.acm.org/about-acm/ about-the-acm-organization

Association for Computing Machinery-ACM. (2018). CC 2001: Curricula Guidelines for Undergraduate Degree Programs in Computer Science. Recuperado de: http://www.acm.org/ education/curricula-recommendations

Association for Computing Machinery-ACM. (2018). CS2008 Curriculum Update: The Computing Curricula Computer Science Volume is complete and approved. Recuperado de: http://www.acm.org/education/curricula-recommendations

Aguilar, W., Alonzo, D., Canul, D. \& Cerón, J. (2012). Percepción social sobre los beneficios de estar viviendo en un área natural protegida: Otoch Máax Yetel Kooh. Teoría y Praxis, (12), 34-51.

Arora, A. \& Gambardella, A. (2004). IT and regional development: lessons from the growth of the software industry in India, Ireland, Israel, Brazil and China. Recuperado de http://www. lse.ac.uk/fmg/research/RICAFE/pdf/RICAFE2-WP60-Arora.pdf

Barchine, G (2006). Una disciplina bio-psico-socio-tecno-cultural. Revista Ingeniería Informáti$c a$, (12). Recuperado de: http://inf.udec.cl/ revista/ediciones/edicion12/articulo\%2012-3.pdf

COLCIENCIAS. (2016). Plan decenal Nacional de Educación. Recuperado de: http://www.plandecenal.edu.co/

DANE. (2007). Portal DANE. Recuperado de: http://www.dane.gov.co/

Revista DINERO. (9 de Octubre de 2016). Revista DINERO. Recuperado de http://www.dinero. $\mathrm{com} /$

ESI. (2008). ESI Main Page. Recuperado de: https:// www.esi-group.com/es 
FEDESOFT. (2018). Página de empleo FEDESOFT. Recuperado de http://fedesoft.org/empleo/?gclid=EAIaIQobChMIjqzZr8Ge1gIVh0OGCh1nvwYgEAAYASAAEgIwf_D_BwE

Heckhausen, H. (1975) Algunos acercamientos a la interdisciplina: disciplina e interdisciplinariedad. En L. Apostel, G. Berger \& G. Michaud, Interdisciplinariedad. Problemas de la enseñanza e investigación en las universidades. Ciudad de México: ANUIES.

Hernández, R., Fernández, C. \& Baptista, P. (1994) Metodología de la investigación. Ciudad de México: McGraw-Hill.

ICEX. (2005). Main page ICEX. Recuperado de: http://www.icex.es/icex/es/index.html

ITEEA. (2016). ITEEA main page. Rrecuperado de https://www.iteea.org

ITEEA. (2018). Standards For Technological Literacy, Technology Education. Recuperado de https://www.iteea.org/39197.aspx

Martínez, S. \& Arango, S. (2015). El crecimiento de la industria del software en colombia: un análisis sistémico. Revista EIA, 12, 95-106.

Martínez, S. \& Arango, S. (2017). Modelo de simulación dinámica para evaluar la inversión en capacidades de innovación tecnológica en la industria colombiana de software. Revista Espacios, 38(9). Recuperado de: http://www. revistaespacios.com/a17v38n09/17380903.html

MEN. (2016). Ministerio de Educación Nacional. Recuperado de http://www.mineducacion.gov. $\mathrm{co} /$

Pérez-González, J., Mateos, C \& Carranza, J. (2009). Polygyny can increase rather than decrease genetic diversity contributed by males relative to females: evidence from red deer. Molecular Ecology, 18(8), 1591-1600.
PROEXPORT. (2010). Informe de exportaciones, inversión extranjera: Nicaragua. Recuperado de http://www.colombiatrade.com.co/sites/default/files/nicaragua.pdf

Repositorio Instintucional de la Universidad Nacional de Colombia. (2016). BDIGITAL Repositorio Institucional UN. Recuperado de http:// www.bdigital.unal.edu.co/

Ruffinatti, A. (9 de Octubre de 2016). La industria del software en la india: ¿un éxito casual?. [Entrada de blog]. Recuperado de: https://winred. com/management/la-industria-del-software-en-la-india-un-exito-casual/gmx-niv116con2774.htm

Villalba, M., Robledo, J. \& Builes, C. (2016). Análisis estratégico de la colaboración entre empresas nacionales y multinacionales de software en Colombia utilizando dinámica de sistemas. International Journal of Psychological Research, 9(1), 83-97. 